\title{
Efectividad del cepillo dental eléctrico versus manual para la remoción de biofilm en pacientes con síndrome de Down.
}

\author{
Effectiveness of electric versus manual toothbrush for \\ biofilm removal in patients with Down syndrome.
}

\author{
Diana Alejandra Torres Tavera,* Miriam Lucía Rocha Navarro, ${ }^{\ddagger}$ Georgina de Jesús Núñez González ${ }^{\S}$
}

\section{RESUMEN}

Introducción: La salud oral en pacientes con síndrome de Down (SD) es un reto, ya que las alteraciones en la motricidad ocasionadas por la discapacidad intelectual (DI) hacen que tareas como la remoción del biofilm oral o placa dentobacteriana (PDB) con el cepillado sea deficiente. La efectividad de los cepillos eléctricos (CE) comparada con los manuales (CM) en afectados con SD sigue siendo un tema debatible ya que no se ha encontrado una homogeneidad de resultados. Es conveniente mayor investigación sobre el tema, debido a que el SD es considerada la alteración cromosómica más frecuente y la causa principal de DI en el mundo. Objetivo: Conocer la efectividad del $\mathrm{CE}$ en comparación con el $\mathrm{CM}$ para la remoción de $\mathrm{PDB}$ en pacientes mexicanos con SD de entre seis y 14 años. Material y métodos: Estudio transversal experimental, con emparejamiento de sujetos con SD de ambos géneros según la edad (seis a 14 años), asignando el cepillo a utilizar (grupo $1 \mathrm{CM}$ o grupo $2 \mathrm{CE}$ ). Se realizó educación dental y método de cepillado con la técnica de Fones, así como control de PDB antes y después el efectuado por medio del índice de O'Leary durante tres días. Se analizaron los resultados utilizando estadística descriptiva e inferencial (prueba de normalidad Lilliefors, prueba t para variables independientes y dependientes). Se aceptó un análisis de significancia $\mathrm{p}$ $<0.05$. Resultados: La utilización de la t para muestras independientes presentó una mejoría en incremento de dicho marcador de O'Leary en el conjunto total en los tres días de seguimiento respectivamente $(\mathrm{t}=6.9$, $\mathrm{p}<0.00002 ; \mathrm{t}=8.4, \mathrm{p}<0.00000 ; \mathrm{yt}=9.5, \mathrm{p}<0.00000)$. Al comparar por prueba $t$ aplicada a muestras dependientes el índice de O'Leary basal versus cada una de las evaluaciones de seguimiento también se observaron diferencias significativas en los dos conjuntos (grupo 1: p

\section{ABSTRACT}

Introduction: Oral health in patients having Down syndrome (DS) is a challenge since the changes in motor skills caused by intellectual disability (ID) deteriorate tasks such as the removal of biofilm or dental plaque (DP) with brushing. The effectiveness of electric toothbrushes (ET) compared to manual toothbrushes (MT) in DS patients remains debatable since no homogeneity of results has been found. Further research on the subject is advisable as DS is considered the most frequent chromosomal alteration and the leading cause of ID in the world. Objective: To know the effectiveness of the ET in contrast to the MT for the removal of DP in Mexican DS patients between six and 14 years old. Material and methods: Experimental cross-sectional study, with the pairing of DS subjects of both genders according to age (six to 14 years), assigning the brush to be used (group 1 MT or group 2 ET). Dental education and brushing were performed employing the Fones technique and DP control before and after brushing employing the O'Leary index for three days. The results were analyzed using descriptive and inferential statistics (Lilliefors normality test, $t$-test for independent and dependent variables). A significance test $p<0.05$ was accepted. Results: The t-test for independent samples showed an improvement in the increase of the O'Leary index in the complete group in the three days of follow-up respectively $(t=6.9, p<0.00002 ; t=$ $8.4, p<0.00000$; and $t=9.5, p<0.00000$ ). When comparing by $t$-test for dependent samples the baseline O'Leary index versus each of the follow-up evaluations, significant differences were also observed in both groups (group 1: $p=0.003, p=0.0006$ and $p=0.0017$; group 2: $p=0.007, p=0.0006$ and $p=0.0002$ ). Conclusion: The dental education and motivation of the patient were decisive for the good

\footnotetext{
* Estudiante de Licenciatura en Odontología.

${ }^{\ddagger}$ Licenciatura en Cirugía Dental, Maestría y Doctorado en Ciencias Médicas, Profesor-investigador.

${ }^{\S}$ Licenciatura en Cirugía Dental, Maestría en Odontología Pediátrica, Profesor.
}

Universidad De La Salle Bajío. León, Guanajuato. México.

Recibido: 26 de mayo de 2021. Aceptado: 18 de julio de 2021.

Citar como: Torres TDA, Rocha NML, Núñez GGJ. Efectividad del cepillo dental eléctrico versus manual para la remoción de biofilm en pacientes con síndrome de Down. Rev ADM. 2021; 78 (4): 189-194. https://dx.doi.org/10.35366/101072 
$=0.003, \mathrm{p}=0.0006$ y $\mathrm{p}=0.0017$; grupo $2: \mathrm{p}=0.007, \mathrm{p}=0.0006$ y $\mathrm{p}=$ 0.0002). Conclusión: La instrucción dental y motivación del paciente fueron determinantes hacia el buen desempeño del cepillado de los dientes y remoción de placa en el par de colectivos, es decir, la reafirmación de la técnica de Fones incrementa gradualmente la mejoría de la forma de uso del cepillo en niños con SD. Ambos métodos con CM y con CE fueron efectivos a fin de remover significativamente la placa, observándose una mayor mejoría en el grupo con $\mathrm{CE}$, sugiriendo que éste disminuye la dificultad de la higiene bucal en personas con SD. Esta línea de investigación es importante en el beneficio de la condición oral de esta población.

Palabras clave: Cepillo eléctrico, cepillo manual, placa dentobacteriana, biofilm, síndrome de Down, índice de O'Leary. performance of tooth brushing and removal of the plaque in both groups. The reaffirmation of the Fones technique gradually increases the improvement of the brushing technique in DS children. Both brushings using $M T$ and ET were effective in removing the biofilm significantly. However, a greater improvement was observed in the group with ET, suggesting that it reduces their difficulty with tooth brushing. This line of research is important to benefit the oral condition of this population.

Keywords: Electric toothbrush, manual toothbrush, dental plaque, biofilm, Down syndrome, O'Leary index.

\section{INTRODUCCIÓN}

$\mathrm{E}$ síndrome de Down (SD), también llamado trisomía 21, es considerado la anormalidad cromosómica más frecuente en el ser humano y la causa principal de discapacidad cognitiva en todo el mundo. ${ }^{1}$ Fue descrito por John Langdon Down en 1861, y se caracteriza por la presencia de un cromosoma extra en el par 21, motivado por falta de disyunción en la meiosis o en la mitosis, o una translocación. ${ }^{2}$

La causa aparente del SD es desconocida, su formación espontánea ha provocado que no existan acciones que puedan impedirlo. Según la OMS, la incidencia estimada a nivel mundial se sitúa en uno de cada 1,000 a 1,100 nacimientos. En México, se ubica en 3.7 por cada 10,000 nacimientos. ${ }^{1}$

Las alteraciones del sistema estomatológico de los pacientes con SD incluyen: lengua fisurada, respiración bucal que provoca sequedad de las mucosas, estomatitis y queilitis angular por emisión constante de saliva, alteración en la erupción dentaria y en el esmalte dental, microdoncia y alta prevalencia de maloclusiones, sobre todo clase III de Angle por insuficiente evolución del maxilar superior y protrusión mandibular, y reacciones desencadenadas por la ingesta de medicamentos (gingivitis, periodontitis). ${ }^{3}$

Además, el paciente con SD presenta una discapacidad, término que abarca deficiencia, limitación de la actividad y restricción de la participación. Por deficiencia se refiere a los problemas que afectan a una estructura o función del cuerpo, disminución de la actividad por las dificultades en la ejecución de acciones o tareas, y por acotación de la intervención o problema para participar en situaciones vitales. ${ }^{4}$ La discapacidad intelectual (DI) afecta su aptitud para aprender y lograr un desarrollo com- pleto de sus capacidades cognitivas, de comunicación, autocuidado, y relaciones interpersonales, influyendo en su adaptación al entorno. ${ }^{5}$

Por causa de esta discapacidad, la higiene oral suele ser un reto frente a los pacientes con SD, sus familiares y odontóloga, debido a que las alteraciones en la motricidad ocasionadas por la DI hacen que tareas como la remoción de placa dentobacteriana (PDB) o biofilm, sea poco efectiva por una destreza manual disminuida, y por consecuencia afectar la salud estomatognática. ${ }^{1}$

El cepillo dental es un aditamento implementado para la eliminación de la PDB que debe limpiar con eficacia y alcanzar todas las superficies dentarias y tejidos blandos de la cavidad oral. ${ }^{6}$ El modelo manual (CM) de cerdas apareció alrededor del año 1600 en China, pero se patentó por primera vez en Estados Unidos (EU) en 1857 y desde entonces ha estado en continua evolución e innovación. En 1960 aparece el primer modelo eléctrico (CE) en EU.7 Se observan dos tipos principales de desplazamiento en un $\mathrm{CE}$, movimiento de oscilación-rotación-pulsación, en el que el cabezal del cepillo (normalmente redondo) se mueve hacia adelante y atrás en un ángulo determinado. El segundo tipo de movimiento es llamado sónico o ultrasónico, generalmente involucra una cabeza de forma rectangular, que se mueve hacia adelante y atrás al girar en un ángulo dado con respecto al eje largo del mango. El diseño del cepillo dental será diferente según el fabricante, cada variante en el invento afectará directamente a su función, pero el objetivo general seguirá siendo el de remover la mayor cantidad de placa microbiana. ${ }^{8}$

Existe poca información acerca de la efectividad del CE en comparación con el CM para la remoción de PDB en pacientes mexicanos de entre seis y 14 años con SD. 


\section{MATERIAL Y MÉTODOS}

Estudio transversal experimental de series cronológicas en personas con SD de ambos géneros, desde seis a 14 años, con DI ligera a moderada y dentición mixta. Se solicitó la autorización de la institución Corazón Down a fin de incluir a los pacientes en la investigación. También los padres o tutores autorizaron la participación de sus hijos por medio de consentimiento informado y todos los seleccionados asintieron su participación en la observación. El protocolo de investigación fue autorizado por el Comité de Ética de ambas instituciones.

Se utilizó una muestra no probabilística por simple disponibilidad, incluyendo al protocolo 10 niños que fueron asignados a dos conjuntos de observación (grupo 1 CM o grupo 2 CE) y a la vez emparejados por edad. A todos los pacientes, con apoyo de sus padres, se les aplicó cuestionario de salud y plática motivacional para alcanzar una correcta técnica de cepillado de Fones, la cual fue practicada y revisada durante una semana. También se evaluó el control de placa (Figura 1) antes y después de realizar el cepillado con CE* $\left({ }^{*}\right.$ Oral B Pro-Salud, Procter and Gamble, México $)$ o $\mathrm{CM}^{¥}\left({ }^{\ddagger}\right.$ Oral B Infantil Stages 4, Procter and Gamble, México) por medio del índice de O'Leary durante tres ocasiones (cada tercer día).

Con la meta de analizar los datos, se utilizó el programa estadístico STATS ${ }^{\mathrm{TM}}$, realizando el examen de normalidad de variables de Lilliefors, así como estadística descriptiva e inferencial (prueba t para muestras independientes buscando valorar discrepancias en el conjunto total y la misma con las muestras dependientes a fin de evaluar diferencias iniciales y de cada una de las evalua-

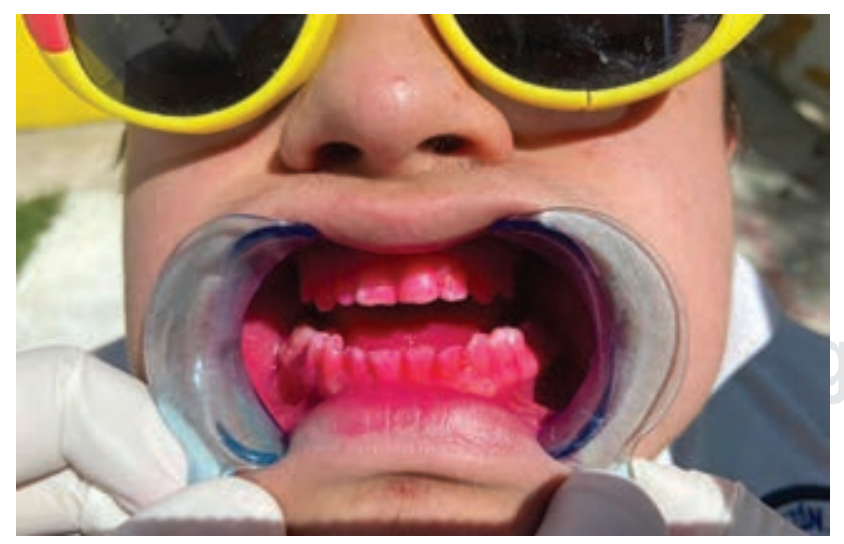

Figura 1: Tinción con solución reveladora de biofilm en un paciente con síndrome de Down.
Tabla 1: Emparejamiento de los sujetos por edad.

Px1: 12 años vs Px6: 12 años

Px2: 13 años vs Px8: 14 años

Px3: 11 años vs Px7: 10 años

Px4: 6 años vs Px10: 7 años

Px5: 9 años vs Px9: 8 años

$\mathrm{Px}=$ paciente

ciones realizadas a el par de conjuntos emparejados). Se consideró significativa una diferencia de $p \leq 0.05$.

\section{RESULTADOS}

De los 10 jóvenes incluidos al estudio, seis fueron masculinos y cuatro femeninos, entre seis y 14 años de edad. La Tabla 1 muestra cómo se emparejaron los pacientes por edad. La edad media fue de 10.2 años dentro de ambos conjuntos.

El cuestionario de salud dental arrojó que la mitad de estos infantes con SD sólo se cepilla los dientes una vez al día, 80\% no ha acudido a revisión odontológica en los últimos dos años y el restante $20 \%$ lo ha hecho solamente debido a urgencias de origen dentario.

Los datos mostraron normalidad. La prueba $\mathrm{t}$ destinada a muestras independientes reveló una diferencia significativa e incrementada del control de la placa comparando la PDB inicial con cada una de las evaluaciones realizadas cada tercer día en el conjunto total; es decir, conforme aumentaba el tiempo de práctica del cepillado de los dientes de los niños con SD, mayor disminución de la placa (Tabla 2).

La prueba t para muestras dependientes emparejó el índice de O'Leary inicial de cada grupo con cada una de las evaluaciones realizadas; se encontraron diferencias significativas entre ellos (Tabla 3).

El emparejamiento de los sujetos por técnica de cepillado dental (CM versus $\mathrm{CE}$ ), no presentó diferencias al inicio o a lo largo de las tres evaluaciones (Tabla 4). El marcador inicial de O'Leary no mostró diferencia basal entre los grupos, por lo que eran equiparables.

\section{DISCUSIÓN}

Los sujetos con SD tienen mayor susceptibilidad a desarrollar problemas periodontales debido a su medicación y falta de destreza manual por DI. Es 
un desafío frente al paciente y familiares lograr una efectiva remoción de la placa oral. Aguirre y colaboradores ${ }^{9}$ evaluaron la aplicación de juegos colectivos adaptados a personas de seis a 32 años con síndrome de Down, como estrategia de intervención educativa en la sanidad bucal, comprobando en pacientes que disminuye considerablemente el índice de higiene oral simplificado (IHOS) después de aplicada la intervención educativa. Por lo que la formación dental, aprendizaje y reforzamiento de técnicas de cepillado sencillas como la de Fones o Stillman modificada con componentes didácticos, así que es imperativo contar con aditamentos innovadores de higiene bucal que les faciliten la eliminación de PDB.

Estos resultados afirman que ambos tipos de cepillos son efectivos en la remoción de la PDB, sin embargo, el grupo con CE presentó una diferencia mayor comparado con el CM. Esto concuerda con las conclusiones de Calvo y coautore ${ }^{10}$ quienes observaron que el CE es más efectivo que el CM para eliminar la placa y disminuir la gingivitis en aquejados por enfermedad periodontal, ya que el sangrado al sondeo fue reducido $44 \%$ en el grupo CE y sólo $37 \%$ en el grupo CM, después de seis meses con respecto a las cifras basales.

También Meléndez y colegas ${ }^{11}$ aseguran que la utilización del CE o el CM en pacientes con SD tiene la capacidad de disminuir la acumulación de PDB, pero el CE demostró una mayor efectividad en la reducción de placa en comparación con CM.

No obstante, dichos resultados difieren de los de Silva y asociados $^{12}$ quienes reportan que el uso de CE o CM no afecta la cantidad de biopelícula dentaria removida en niños y adolescentes con SD, ni influye en su cooperación durante el procedimiento. Esta discrepancia sugiere que los infantes con SD y sus padres no fueron motivados adecuadamente hacia lograr una efectiva eliminación de placa.

A pesar de que con el cuestionario de salud previo a la educación dental, aprendizaje de la técnica de Fones y estimulación al paciente y sus familiares, se demostró una frecuencia de cepillado de sólo una vez al día en la mitad de los pacientes, estos hábitos cambiaron debido

Tabla 2: Diferencias entre el índice de O’Leary inicial versus grupo total con prueba t para muestras independientes.

\begin{tabular}{lcccc} 
& Media* & Media* & t & \\
\hline Índice de O'Leary vs evaluación 1 & $86.1 \pm 13.5$ & $44.7 \pm 13.3$ & 6.9 & 0.000002 \\
Índice de O'Leary vs evaluación 2 & $86.1 \pm 13.5$ & $36.6 \pm 12.8$ & 8.4 & 0.000000 \\
Índice de O'Leary vs evaluación 3 & $86.1 \pm 13.5$ & $30.2 \pm 12.6$ & 9.6 & 0.000000 \\
\hline
\end{tabular}

* Media \pm desviación estándar. Índice de O’Leary en \%.

\begin{tabular}{|c|c|c|c|c|c|c|}
\hline \multirow[b]{2}{*}{ Índice de O’Leary } & \multicolumn{3}{|c|}{ Grupo 1} & \multicolumn{3}{|c|}{ Grupo 2} \\
\hline & Media* & $\mathrm{t}$ & $\mathrm{p}$ & Media* & $\mathrm{t}$ & p \\
\hline Inicial & $82.6 \pm 16.31$ & & & $89.6 \pm 10.71$ & & \\
\hline Evaluación 1 & $47.4 \pm 8.23$ & 6.13 & 0.003 & $42.0 \pm 17.64$ & 5.02 & 0.007 \\
\hline Inicial & $82.6 \pm 16.31$ & & & $89.6 \pm 10.71$ & & \\
\hline Evaluación 2 & $42.6 \pm 13.97$ & 9.87 & 0.0006 & $30.6 \pm 9.07$ & 9.84 & 0.0006 \\
\hline Inicial & $82.6 \pm 16.31$ & & & $89.6 \pm 10.71$ & & \\
\hline Evaluación 3 & $37.2 \pm 13.53$ & 7.40 & 0.0017 & $23.2 \pm 7.04$ & 12.31 & 0.0002 \\
\hline
\end{tabular}


Tabla 4: Grupos emparejados con prueba t para muestras dependientes.

\begin{tabular}{|c|c|c|c|c|}
\hline \multirow[b]{2}{*}{ Índice de O’Leary } & \multirow{2}{*}{$\begin{array}{l}\text { Grupo } 1 \\
\text { Media* }\end{array}$} & \multicolumn{3}{|c|}{ Grupo 2} \\
\hline & & Media* & $\mathrm{t}$ & $\mathrm{p}$ \\
\hline Inicial & $82.6 \pm 16.3$ & $89.6 \pm 10.7$ & -74 & 0.5 \\
\hline Evaluación 1 & $47.4 \pm 8.2$ & $42.0 \pm 17.6$ & -75 & 0.5 \\
\hline Evaluación 2 & $42.6 \pm 13.9$ & $30.6 \pm 9.1$ & 2.19 & 0.09 \\
\hline Evaluación 3 & $37.3 \pm 13.5$ & $23.2 \pm 7.0$ & 1.9 & 0.12 \\
\hline
\end{tabular}

a que se concientizaron acerca de la importancia del autocuidado de los dientes, atención con el apoyo de los parientes, y logrando que el uso del cepillo dentario se convierta en una tarea cotidiana en casa y escuela. Además, se reforzó la trascendencia de acudir a revisiones odontológicas periódicas preventivas y así evitar urgencias de origen dental.

Las limitaciones del estudio fueron un tamaño de la muestra pequeño y realizar un seguimiento de control de PDB de manera longitudinal procurando verificar si se mantiene el control de placa y la motivación a largo plazo.

\section{CONCLUSIONES}

La motivación y actitud colaborativa del paciente fue determinante en el buen desempeño del cepillado dentario. La práctica constante de dicha rutina, al igual que la supervisión por un adulto, mejoran el control del biofilm en pequeños con SD sin importar el cepillo dental utilizado.

La técnica de Fones con cepillo eléctrico en niños y adolescentes con síndrome de Down es un mejor tratamiento para remover la placa que el modelo manual, lo que sugiere que en personas con discapacidad cognitiva y motora es la alternativa ideal, con tal de superar la falta de destreza psicomotora. Es conveniente el seguimiento de esta línea de investigación en sujetos con síndrome de Down, mejorando así la calidad de vida de esta población.

\section{AGRADECIMIENTOS}

Un agradecimiento especial al Centro Psicoeducativo Corazón Down A.C. por brindar todas las facilidades para realizar esta investigación.

\section{REFERENCIAS}

1. Delgado-Arteaga A, Reyna-Márquez M, Murillo-Flores V, LópezGonzález A, Rodríguez-Paniagua O, Gómez-Castañón G et al. Manejo odontológico en paciente con Síndrome de Down. Revista Latinoamericana de Ortodoncia y Odontopediatría. 2019.

2. Sierra Romero MC, Navarrete Hernández E, Canún Serrano $S$, Reyes Aldelmo PE, Valdés Hernández J. Prevalencia del síndrome de Down en México utilizando los certificados de nacimiento vivo y de muerte fetal durante el periodo 2008-2011. Bol Med Hosp Infant Mex. 2014; 71 (5): 292-297.

3. Zuluaga M, Martínez C, Cardona N, Gutiérrez L, Giraldo A, Jiménez M. Manejo de la salud bucal en discapacitados. Rev CES Odont. 2017; 30 (2): 23-36.

4. Organización Mundial de la Salud. Discapacidad y Salud [en línea]. OMS; 2018 [fecha de acceso 1 de octubre de 2020]. Disponible en: https://www.who.int/es/news-room/fact-sheets/detail/disabilityand-health

5. Peredo Videa RA. Comprendiendo la discapacidad intelectual:: datos, criterios y reflexiones. Rev Invest Psicol. 2016; (15): 101-122.

6. Newman MG, Takei HH, Klokkevold PR, Carranza FA. Periodonto logia clínica de Carranza. 13a edición. USA: Elsevier; 2019.

7. Nápoles González IJ, Fernández Collazo ME, Jiménez Beato P. Evolución histórica del cepillo dental. Rev Cubana Estomatol. 2015; 52 (2): 208-216

8. Márquez JC, Lacruz RR. Aspectos morfológicos y psicológicos en el diseño de cepillos dentales. PORTAFOLIO. 2004; 1 (9): 16A-24A.

9. Aguirre J, Porras D, Ríos K. Estrategia de intervención educativa sobre la salud bucal en pacientes de 6 a 32 años con síndrome de Down. Rev Estomatol Herediana. 2015; 25 (4): 262-267.

10. Calvo JC. Cepillos dentales oscilantes-rotacionales. Pulsátiles: evidencia científica. RCOE. 2015; 20 (Supl 1).

11. Meléndez D. Evaluación de la eficacia en la remoción de biopelícula dental mediante el uso de cepillo manual y eléctrico en niños con síndrome de Down [Tesis de Licenciatura]. Ecuador: Universidad de las Américas; 2016.

12. Silva AM, Miranda LFB, Araujo ASM, Prado Junior RR, Mendes RF. Electric toothbrush for biofilm control in individuals with Down syndrome: a crossover randomized clinical trial. Braz Oral Res. 2020; 34:e057. doi: 10.1590/1807-3107bor-2020.vol34.0057. PMID: 32578800. 
Correspondencia:

Diana Alejandra Torres Tavera

E-mail: dtorrestavera@gmail.com

Conflicto de intereses: No existe conflicto de intereses. Aspectos éticos: Se realizó un convenio entre los directores de ambas instituciones, se informó a los pacientes y sus tutores el propósito del estudio y los procedimientos a realizar durante éste. Se autorizó la participación por medio del consentimiento informado, todos los pacientes asintieron con la cabeza. El protocolo fue revisado por la Dra. Miriam Lucía Rocha Navarro, docente de la Facultad de Odontología de la Universidad De La Salle Bajío (UDLSB). Financiamiento: La primera autora absorbió el financiamiento total de la investigación. 